\title{
Diffusion-Weighted Imaging with Two Different $b$-Values in Detection of Solid Focal Liver Lesions
}

\author{
Da-wei Yang, ${ }^{1,2}$ Ke-yang Wang, ${ }^{1}$ Xun Yao, ${ }^{3}$ Hui-yi Ye, ${ }^{4}$ Tao Jiang, ${ }^{5}$ Yuan Liu, ${ }^{6}$ Jia-yin Gao, \\ Min Chen, ${ }^{7}$ Cheng Zhou, ${ }^{7}$ and Zheng-han Yang ${ }^{1,2}$ \\ ${ }^{1}$ Department of Radiology, Beijing Friendship Hospital, Capital Medical University, Beijing 100050, China \\ ${ }^{2}$ National Clinical Research Center for Digestive Diseases, Beijing 100050, China \\ ${ }^{3}$ Department of Radiology, Peking University People's Hospital, Beijing 100044, China \\ ${ }^{4}$ Department of Radiology, PLA General Hospital, Beijing 100853, China \\ ${ }^{5}$ Department of Radiology, Beijing Chaoyang Hospital, Capital Medical University, Beijing 100020, China \\ ${ }^{6}$ Department of Ultrasound, Beijing Hospital, Beijing 100730, China \\ ${ }^{7}$ Department of Radiology, Beijing Hospital, Beijing 100730, China
}

Correspondence should be addressed to Zheng-han Yang; zhenghanyang@263.net

Received 22 September 2015; Revised 19 November 2015; Accepted 29 November 2015

Academic Editor: Yi-Xiang Wang

Copyright (C) 2016 Da-wei Yang et al. This is an open access article distributed under the Creative Commons Attribution License, which permits unrestricted use, distribution, and reproduction in any medium, provided the original work is properly cited.

One hundred and eighty-two consecutive patients with suspected liver disease were recruited to receive diffusion-weighted imaging (DWI) with two different $b$-values, in comparison with T2-weighted imaging (T2WI). The detection rate of three MR sequences in solid focal liver lesions (FLLs) and subgroup analyses were performed. Our prospective study found that DWI600 was equivalent to DWI100 and T2WI for the detection of solid FLLs overall but was significantly more accurate in the detection of malignant solid FLLs and lesions larger than $10 \mathrm{~mm}$.

\section{Introduction}

Early detection and diagnosis of hepatic tumor are an important step in clinical work, which would allow effective surgical or mini-invasive therapy [1-6]. With the advances in magnetic resonance imaging (MR) technology, diffusionweighted magnetic resonance imaging (DWI) is now widely used as a standard imaging sequence in clinical work and shows its potential benefit in evaluation of the focal hepatic tumor [7-11]. DWI with small $b$-values less than 100$150 \mathrm{sec} / \mathrm{mm}^{2}$ can suppress the intrahepatic vascular signal, creating the so-called black blood effect, which improves the detection of small focal liver lesions (FLLs) especially localized near small hepatic vessels. Meanwhile, DWI with low $b$-value has higher imaging quality compared with single shot fast spin-echo sequences [11-13], due to the fact that it is less affected by artifacts such as eddy currents or blurring.

A substantial number of studies [14-17] have compared low $b$-value DWI with T2-weighted imaging (T2WI) for image quality and detection of FLLs. These studies generally showed better performance of DWI with low $b$-value in terms of lesion detection and conspicuity compared with T2WI. However, most previous studies mainly focused on the detection of metastases or just included cysts and hemangiomas as benign lesions, lacking of common solid FLLs such as focal nodular hyperplasias (FNHs), or other less common solid FLLs such as inflammatory pseudotumors (IPTs). Furthermore, DWI with low $b$-value could not simultaneously improve the detection as well as characterization of lesion, which is usually performed with DWI with higher $b$-value $\left(b>500 \mathrm{sec} / \mathrm{mm}^{2}\right)$ and/or other conventional sequences.

DWI with higher $b$-value mainly reflects diffusion information of water molecules motion within the lesions, which help to improve the characterization of solid FLLs [8]. Meanwhile, we found in practice that DWI with higher $b$ value also enables a better detection of lesions in liver or pancreas compared with T2WI or other conventional sequences. For example, solid FLLs such as FNHs and hepatocellular carcinomas (HCCs) sometime can be difficult to be detected on T2WI or even DWI with low $b$-value due to either iso- or 
TABLE 1: Parameters of three evaluation MR sequences.

\begin{tabular}{lccc}
\hline Parameters & DWI600 & DWI100 & T2WI \\
\hline Acquisition mode & Respiratory-triggered & Breath-hold & Respiratory-triggered \\
Repetition time (TR) & 2-3 respiratory cycles & $2400 \mathrm{~ms}$ & $2-3$ respiratory cycles \\
Echo time (TE) & Minimum & Minimum & $80 \pm 10 \mathrm{~ms}$ \\
$b$-value $\left(\mathrm{s} / \mathrm{mm}^{2}\right)$ & 0 and 600 & 0 and 100 & $/$ \\
Section thickness $(\mathrm{mm})$ & 6 & 6 & 6 \\
Intersection gap $(\mathrm{mm})$ & 1.5 & 1.5 & 1.5 \\
Field of view (mm) & $320-380$ & $320-380$ & $320-380$ \\
Rectangle FOV & $90 \%-100 \%$ & $128 \times 128$ & $75 \%$ \\
Matrix & $128 \times 128$ & 2 & $224 \times 320$ \\
Number of signal averages & 4 & 2 & 2 \\
Parallel acceleration factor & 2 & 64 & 1 \\
Echo train length & 64 & $19 \mathrm{~s}$ & $3-5$ min \\
Acquisition time & $2-3$ min & 20 \\
\hline
\end{tabular}

DWI600: diffusion-weighted imaging with $b$-value of $0,600 \mathrm{sec} / \mathrm{mm}^{2}$; DWI100: diffusion-weighted imaging with $b$-value of $0,100 \mathrm{sec} / \mathrm{mm}^{2}$; T2WI: T2weighted imaging.

slightly hypersignal intensity to liver parenchyma [18-20]; however, those lesions could be more conspicuous on DWI with higher $b$-value. Although some studies [16, 21-23] have investigated DWI with higher $b$-value in FLL detection, none of the studies has discussed the role of DWI with higher $b$ value in detection of solid FLLs covering the topic of common disease. Therefore, the purpose of this multicenter clinical study was to prospectively investigate the DWI with low $b$ values of $0,100 \mathrm{sec} / \mathrm{mm}^{2}$ (DWI100), DWI with higher $b$-value of $0,600 \mathrm{sec} / \mathrm{mm}^{2}$ (DWI600) in detection of solid FLLs in a large number of patients with a wide spectrum of lesions, in comparison with T2WI.

\section{Methods and Materials}

2.1. Patients. This prospective, multicenter study was approved by Institutional Human Ethics Board and registered in http://www.chictr.org.cn/ numbering ChiCTR-DDT11001587. Written informed consent was obtained from all patients. From June 2011 to December 2012, 182 patients were recruited in 6 hospitals (Beijing Friendship Hospital, Beijing Hospital, PLA General Hospital, Beijing Chaoyang Hospital, Nantong Third People's Hospital, and Ningbo Lihuili Hospital). The inclusion criteria were patients who have (1) focal liver lesions (FLLs) found on ultrasound or CT, (2) chronic liver disease or cirrhosis or viral hepatitis $\mathrm{B} /$ hepatitis C infection, (3) extrahepatic malignancy, and (4) no contraindication to MR contrast agents. 85 patients were excluded according to at least one of the following exclusion criteria: (1) undergoing antineoplastic treatment before MR scanning $(n=8),(2)$ no definite diagnosis $(n=6)$, (3) incomplete DW imaging $(n=3)$, (4) no lesion detected $(n=37)$, and (5) only cystic lesion detected $(n=31)$. Those without histologic diagnosis as well as typical MR findings finally were defined as patients with no definite diagnosis, who were then strongly suggested to take follow-up under the guidance of doctor considering other useful clinical tests. Patients with incomplete DW imaging were excluded from the study population; however, the lack of several DW images did not hamper making diagnosis.

2.2. MR Imaging. A $1.5 \mathrm{~T}$ MRI whole-body scanner (Signa Twin-speed HD, GE Healthcare, Milwaukee, WI) with an eight-element phased array coil was used for signal reception in all study sites. Gradient strengths were $23 / 40 \mathrm{mT} / \mathrm{m}$. Gradient slew rate were $80 / 150 \mathrm{mT} / \mathrm{m} / \mathrm{ms}$. Diffusion-weighted MR imaging with low and higher $b$-value sequences and respiratory-triggered T2-weighted fast spin-echo imaging were performed as study MR sequences. The spectrally selective fat suppression technique was used at all three MR sequences. The detailed parameters for three MR sequences are shown in Table 1 . We choose the $b$-value of $100 \mathrm{~s} / \mathrm{mm}^{2}$ and $600 \mathrm{~s} / \mathrm{mm}^{2}$ to represent the low and higher $b$-value, respectively, because both of them were used routinely in our clinical work and proven to be a good option.

Other MR sequences including in- and opposed-phase spoiled gradient-recalled echo T1-weighted imaging and contrast-enhanced fat-suppressed three-dimensional spoiled gradient-recalled echo imaging were performed.

2.3. Reference Standard. Standard of reference for FLL detection was established by the consensus reading of the two observers (Ye Tan and Jie Zhu, with 15 and 10 years of experience in abdominal imaging, resp.), using all available MR sequences including precontrast T1-weighted sequence, in- and opposed-phase gradient-recalled-echo T1-weighted sequence, and dynamic contrast-enhanced MR sequence. A lesion was considered as present if it could be detected on at least one sequence and also was confirmed by histopathologic analysis or follow-up MR imaging.

FLLs characterization was established optimally by histopathologic findings. For cases without available histopathologic findings, the clinical diagnosis was made by the combination of clinical history, typical MR imaging findings, and follow-up MR imaging with a minimum interval of 6 months. The clinical diagnosis of benign lesions 
including FNH, IPT, solitary necrotic nodule (SNN), and hepatic pseudolipoma was made by using validated criteria [24-27] and by their stable appearance at follow-up MR imaging with a minimum interval of 6 months. HCCs were diagnosed clinically from a complicated consideration of cirrhosis background, typical imaging findings [28, 29], the American Association for the Study of Liver Disease (AASLD) criteria for HCC [30], elevated tumor markers (e.g., $\alpha$-fetoprotein), and progressively enlargement in follow-up. Metastases were diagnosed on the basis of presence of a known primary malignancy, MR imaging findings [31, 32], and follow-up imaging results showing interval progression.

2.4. Qualitative Evaluation. All MR images were independently interpreted by two observers (Yue Guo and Chen Zhang, with 8 and 7 years of experience in abdominal imaging, resp.) who were blinded to clinical history and imaging reports. DWI600, DWI100, and T2WI were randomly analyzed in three sessions separated by at least 3 weeks to minimize a recall bias. All the cases in each session were interpreted in a random manner. For each patient, the number, size, location (with Couinaud segments delineated), and image number of FLLs were recorded. A maximum of 5 largest lesions were recorded per patient, if multiple FLLs were present. Evaluation was done at GE ADW 4.4 workstation. Each sequence for lesion conspicuity was subjectively rated by using a four-point scale, as follows: score 1, definitely not present; score 2, probably not present; score 3 , probably present; score 4 , definitely present. Positive detection was calculated based on lesions assigned more than or equal to score 3.

2.5. Statistical Analysis. The diagnostic accuracy of DWI600, DWI100, and T2WI for solid FLLs detection was evaluated by comparing the detection rate between each two MR sequences. A statistical analysis was done by using a binary logistic regression model in which the detection accuracy was included as a dependent variable. Subgroup analyses based on the type (benign and malignant), size ( $\leq 10 \mathrm{~mm},>10 \mathrm{~mm})$, and location (right lobe and left lobe) were also performed. The cut-point of $10 \mathrm{~mm}$ selected was based on the average diameter of intrahepatic vessels we measured.

$k$ statistic was used to assess interobserver agreement for lesion detection, defined as poor $(<0.2)$, fair $(0.21-0.40)$, moderate (0.41-0.60), good (0.61-0.80), and excellent (0.81$1.00)$ agreement. All statistical analyses were performed using SPSS 17.0 software (Windows, SPSS, Chicago, IL). A $P$ value of less than 0.05 was considered to be statistically significant.

\section{Results}

3.1. Consensus Reading. Ninety-seven patients had a total of 137 solid FLLs (size range, 5.5-75 mm; mean, $21.9 \mathrm{~mm}$ ) (Table 2). Sixty patients had 96 malignant solid FLLs, including 73 HCCs, 20 metastases, two cholangiocarcinomas, and one hemangioendothelioma. Histopathologic diagnosis was used for 52 HCCs, one metastatic lesion, two cholangiocarcinomas, and one hemangioendothelioma. Clinical diagnosis was used for $21 \mathrm{HCCs}$ and 19 metastases. Thirty-seven
TABLE 2: Clinical information of the 97 patients and characteristics of 137 solid FLLs.

\begin{tabular}{ll}
\hline Age range (mean age) & $25-77$ years (52.2 years) \\
Sex $(\mathrm{M} / \mathrm{F})$ & $65 / 32$ \\
& 30 chronic hepatitis \\
& 25 liver cirrhosis \\
Background liver & 20 steatosis \\
& 22 normal liver \\
Diagnosis of the lesions & \\
$(n=137)$ & 14 FNHs \\
& 10 IPTs \\
& 9 SNNs \\
& 5 hepatic pseudolipomas \\
Benign $(n=41)$ & 1 angiomyolipoma \\
& 1 hepatic adenoma \\
& 1 ectopic adrenal adenoma \\
& 73 HCCs \\
& 20 metastases \\
& 2 cholangiocarcinomas \\
& 1 hemangioendothelioma \\
Malignant $(n=96)$ & 41 left lobes and 96 right lobes \\
& 3 rectal-colons \\
Location of the lesions & 1 breast \\
Primary site of malignancy & 1 pancreas \\
Patients $(n=5)$ &
\end{tabular}

FNH: focal nodular hyperplasia; IPT: inflammatory pseudotumor; SNN: solitary necrotic nodule; HCC: hepatocellular carcinoma.

patients had 41 benign solid FLLs, including 14 FNHs, 10 IPTs, nine SNNs, five hepatic pseudolipomas, one angiomyolipoma, one hepatic adenoma, and one ectopic adrenal adenoma. Among those benign solid FLLs, one angiomyolipoma, two FNHs, one adenoma, one ectopic adrenal adenoma, two IPTs, and one hepatic pseudolipoma were diagnosed pathologically. The diagnosis of remaining benign solid FLLs was made clinically according to the standard of reference.

3.2. Qualitative Evaluation. The detection rates using different MR sequences by each observer were shown in Table 4. Interobserver agreement for the FLLs detection using DWI600, DWI100, and T2WI was excellent, given the $k$ values of $0.982,0.900$, and 0.861 , respectively.

The subgroup analysis by lesion size showed that three MR sequences were more accurate in detection of lesions $(>10 \mathrm{~mm})$ than of lesions smaller than $10 \mathrm{~mm}(P<0.001$, for all MR sequences) (Table 5). However, there were no significant differences between each two MR sequences in detection of lesion no matter larger or smaller than $10 \mathrm{~mm}$.

The subgroup analysis by lesion location showed there were no significant differences between each two MR sequences in detection of lesions in left lobe or in right lobe (Table 5). However, T2WI was significantly better in detection of FLLs in the left lobe than in the right lobe $(76.8 \%$ versus $56.3 \%, P<0.05)$.

3.3. Missed FLLs. The missed FLLs by both observers at DWI600 included 13 HCCs, eight FNHs, five pseudolipomas, six IPTs, two metastases, four SNNs, and one hepatic adenoma. 
TABLE 3: Detection rate of solid FLLs with each sequence in all, benign, and malignant lesions.

\begin{tabular}{llccc}
\hline Sequence & & All lesions $(n=137)$ & Benign lesions $(n=41)$ & Malignant lesions $(n=96)$ \\
\hline DWI600 & & $71.1(97.5 / 137)$ & $40.2(16.5 / 41)$ & $84.4(81 / 96)$ \\
DWI100 & $67.9(93 / 137)$ & $57.3(23.5 / 41)$ & $72.4(69.5 / 96)$ \\
T2WI & & $62.4(85.5 / 137)$ & $42.7(17.5 / 41)$ & $70.8(68 / 96)$ \\
& DWI100 versus DWI600 & 0.573 & 0.127 & 0.04 \\
$P$ value & DWI100 versus T2WI & 0.333 & 0.190 & 0.838 \\
& DWI600 versus T2WI & 0.125 & 0.825 & 0.024 \\
\hline
\end{tabular}

Data are averaged for two independent observers. Unless otherwise indicated, numbers are percentages, with raw data in parentheses. DWI600: diffusionweighted imaging with $b$-value of $0,600 \mathrm{sec} / \mathrm{mm}^{2}$; DWI100: diffusion-weighted imaging with $b$-value of $0,100 \mathrm{sec} / \mathrm{mm}^{2}$; T2WI: T2-weighted imaging.

TABLE 4: Detection rate of solid FLLs with each sequence by the two readers in all, benign, and malignant lesions.

\begin{tabular}{ccccr}
\hline & Sequence & All lesions $(n=137)$ & Benign lesions $(n=41)$ & Malignant lesions $(n=96)$ \\
\hline \multirow{3}{*}{ Observer 1 } & DWI600 & $71.5(98 / 137)$ & $41.4(17 / 41)$ & $84.4(81 / 96)$ \\
& DWI100 & $69.3(95 / 137)$ & $60.9(25 / 41)$ & $72.9(70 / 96)$ \\
Observer 2 & T2WI & $59.1(81 / 137)$ & $36.6(15 / 41)$ & $68.8(66 / 96)$ \\
& DWI600 & $70.8(97 / 137)$ & $39.0(16 / 41)$ & $84.4(81 / 96)$ \\
& DWI100 & $66.4(91 / 137)$ & $53.7(22 / 41)$ & $71.9(69 / 96)$ \\
& T2WI & $65.7(90 / 137)$ & $48.8(20 / 41)$ & $72.9(70 / 96)$ \\
\hline
\end{tabular}

Unless otherwise indicated, numbers are percentages, with raw data in parentheses. DWI600: diffusion-weighted imaging with $b$-value of $0,600 \mathrm{sec} / \mathrm{mm}^{2}$; DWI100: diffusion-weighted imaging with $b$-value of $0,100 \mathrm{sec} / \mathrm{mm}^{2}$; T2WI: T2-weighted imaging.

TABLE 5: Detection rate of solid FLLs with each sequence stratified by location and size.

\begin{tabular}{llcccc}
\hline \multirow{2}{*}{ Sequence } & & \multicolumn{2}{c}{ Lesion location } & \multicolumn{2}{c}{ Lesion size } \\
& & Left lobe $(n=41)$ & Right lobe $(n=96)$ & $\leq 10 \mathrm{~mm}$ & \multicolumn{1}{c}{$>10 \mathrm{~mm}$} \\
\hline DWI600 & & $79.3(32.5 / 41)$ & $67.7(65 / 96)$ & $31.2(7.5 / 24)$ & $79.6(90 / 113)$ \\
DWI100 & & $70.7(29 / 41)$ & $66.7(64 / 96)$ & $25(6 / 24)$ & $77.0(87 / 113)$ \\
T2WI & & $76.8(31.5 / 41)$ & $56.3(54 / 96)$ & $16.7(4 / 24)$ & $72.1(81.5 / 113)$ \\
& DWI100 versus DWI600 & 0.411 & 0.878 & 0.588 & 0.628 \\
$P$ value & DWI100 versus T2WI & 0.573 & 0.138 & 0.477 & 0.212 \\
& DWI600 versus T2WI & 0.794 & 0.102 & 0.175 \\
\hline
\end{tabular}

Data are averaged for two independent observers. Unless otherwise indicated, numbers are percentages, with raw data in parentheses. DWI600: diffusionweighted imaging with $b$-value of $0,600 \mathrm{sec} / \mathrm{mm}^{2}$; DWI100: diffusion-weighted imaging with $b$-value of $0,100 \mathrm{sec} / \mathrm{mm}^{2}$; T2WI: T2-weighted imaging.

The missed FLLs by both observers at DWI100 included 20 HCCs, five FNHs, five pseudolipomas, four IPTs, four metastases, one SNN, one hepatic adenoma, and one cholangiocarcinoma.

The detection rates using different MR sequences in all, benign, and malignant solid FLLs were shown in Table 3. The overall detection rate in all solid FLLs with DWI600, DWI100, and T2WI were $71.1 \%$, 67.9\%, and $62.4 \%$, respectively. There were no significant differences between each two MR sequences. For malignant solid FLLs, DWI600 allowed identification of more FLLs $(84.4 \%)$ than DWI100 (versus $72.4 \%, P<0.05$ ) and T2WI (versus 70.8\%, $P<0.05$ ) (Figures 1 and 2). The subgroup analysis in benign solid FLLs showed that the detection rate of DWI100 (57.3\%) was higher than DWI600 (40.2\%) and T2WI (42.7\%) without significant difference (Figure 3). Meanwhile, DWI600 and T2WI were more accurate in the detection of malignant solid FLLs than of benign lesions (84.4\% versus $40.2 \%, P<0.001 ; 70.8 \%$ versus $42.7 \%, P<0.05$, resp.). However, no significant difference was observed for DWI100 in the detection of malignant solid FLLs in comparison of benign lesions $(P=$ 0.082).

The missed FLLs by both observers at T2WI included 22 HCCs, seven FNHs, four pseudolipomas, four IPTs, four metastases, five SNNs, and one hepatic adenoma.

\section{Discussion}

Our study showed that there were no significant differences among DWI600 (71.1\%), DWI100 (67.9\%), and T2WI (62.4\%) in detection of solid FLLs overall. However, DWI600 (84.4\%) was significantly better than DWI100 (versus 72.4\%, $P<$ 0.05 ) and T2WI (versus $70.8 \%, P<0.05$ ) in detection of malignant solid FLLs. It is well known that DWI with low $b$-value of approximately $100-150 \mathrm{sec} / \mathrm{mm}^{2}$ is hypothesized to attenuate signal from microcirculatory perfusion, while DWI with higher $b$-value $\left(b>500 \mathrm{~s} / \mathrm{mm}^{2}\right.$ ) is thought to reveal restriction of water molecular diffusion in lesions [1113]. With the highly cellular tissue, the tortuosity of the extracellular space, and the high density of cell membranes, 


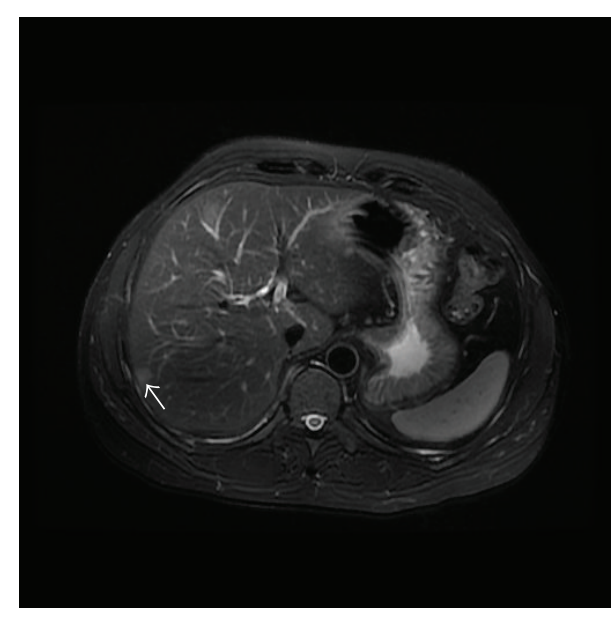

(a)

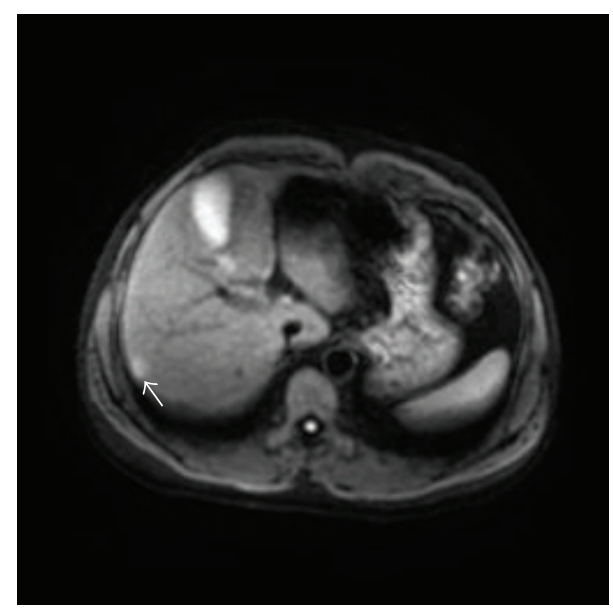

(c)

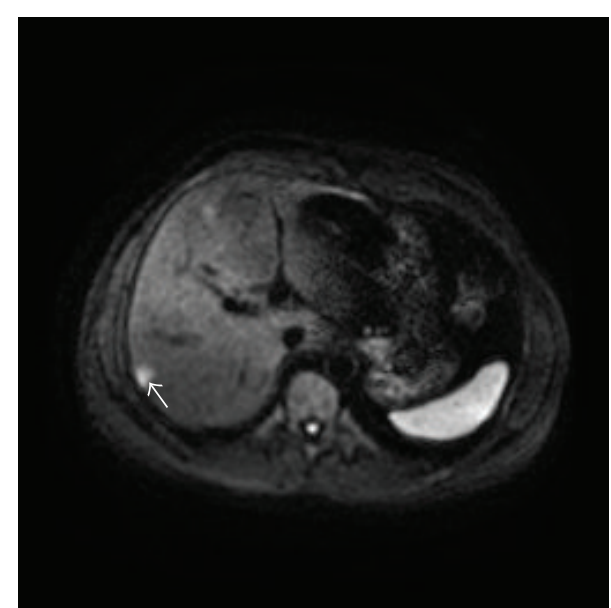

(b)

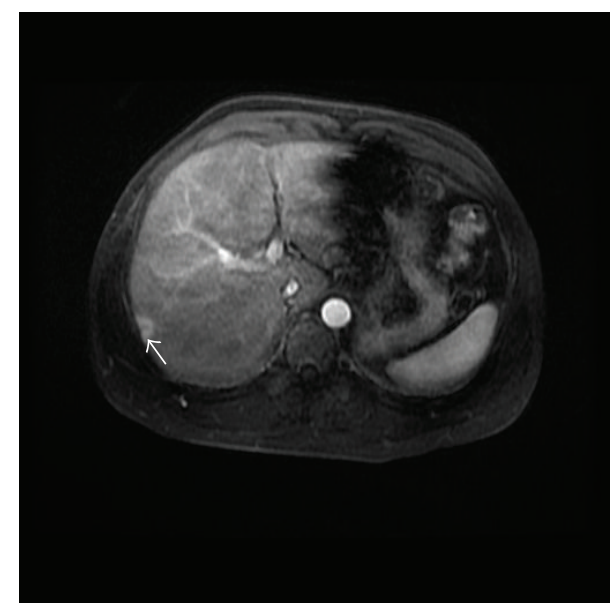

(d)

Figure 1: Transverse images in 49-year-old man with HCC. (a) Fat-suppressed T2-weighted fast spin-echo image shows that lesion is slightly hyperintense to the liver. (b) DW image with higher $b$-value shows that lesion is strongly hyperintense to the liver. (c) DW image with low $b$-value shows that lesion is slightly hyperintense to the liver with ill-defined margin. (d) Contrast-enhanced T1-weighted image in arterial phase demonstrates strong enhancement of lesion.

malignant FLLs can be more easily detected on DWI600 than on DWI100. In addition, T2WI was poorly reliable in detection of malignant solid FLLs when it showed iso- or slightly hypersignal intensity to liver parenchyma. Several studies $[18,33]$ have shown the limitation of T2WI in the detection of HCC in cirrhosis, mostly related to HCC signal intensity on T2WI images with appearing iso- or hypointense in $42.1 \%-53 \%$ of HCC. Results of our studies were in line with the findings of previous reports with lower rate of $32.2 \%$ (23.5/73, average between two observers) HCCs defined as undetectable (score < 3 ) according to the subjective rate system on T2WI, in comparison with $17.8 \%$ (13/73, average between two observers) HCCs undetectable on DWI600.

In addition, our study showed that DWI600 (40.2\%) and T2WI (42.7\%) were inferior in detecting benign solid FLLs in comparison with DWI100 (57.3\%), although the differences did not reach significance. In our study, four FNHs, three SNNs, and two IPTs by observer 1 and three FNHs, three SNNs, and one IPT by observer 2 were visible on DWI100 but not on DWI600. The difference between DWI100 and DWI600 in detection of benign lesions is possibly attributed to three factors. First, over half of benign solid FLLs in our study did not show diffusion restriction. Second, although some benign solid lesions showed high signal intensity on DWI600 due to restricted water molecular diffusion, the lesion-to-liver contrast of those lesions on DWI600 was not as high as malignant lesions such as HCC [34]. Third, black blood effect, better imaging quality, and better contrast-tonoise ratio with DWI100 result in the better lesion conspicuity $[12,13]$. Unfortunately, not enough benign solid FLLs as much as malignant lesions were included in our study; the potential benefit of DWI100 in detection of benign solid FLLs needs more studies to be investigated.

Of interest, our study found DWI600 had the higher detection rate of FLLs on left lobe than on right lobe. Several studies $[21,35]$ reported the poor visibility of the left lobe on DWI due to cardiac-motion induced signal loss; DWI was thought to be less sensitive in the left lobe. However, one must 


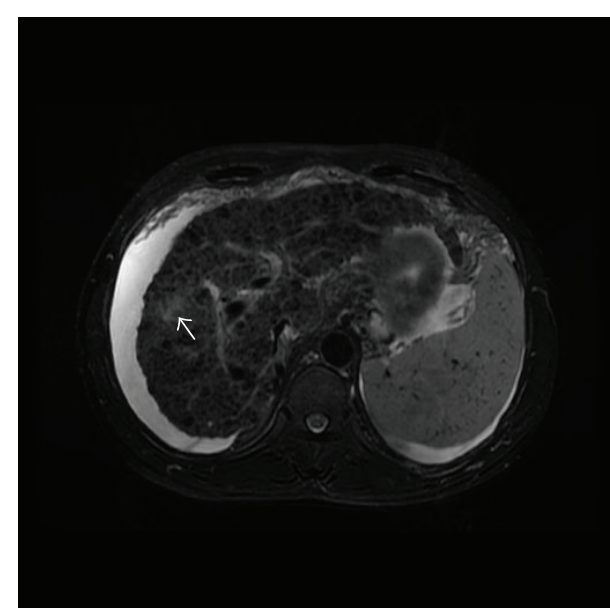

(a)

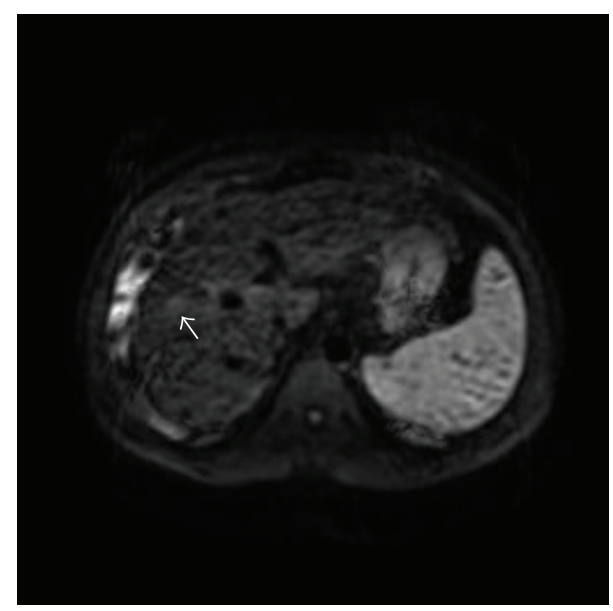

(c)

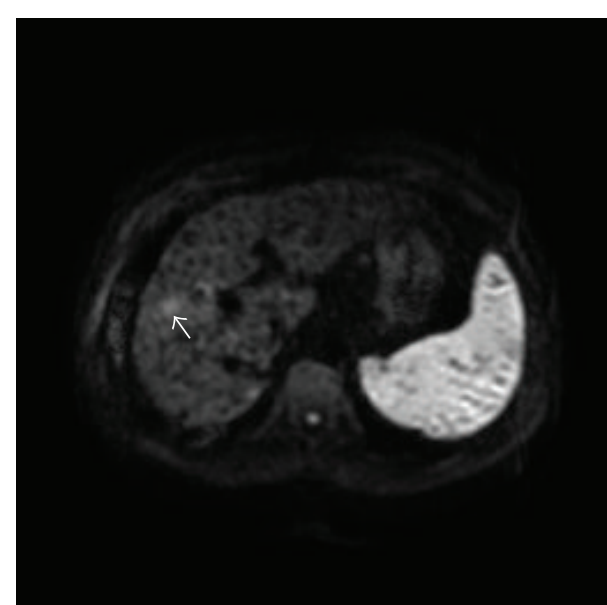

(b)

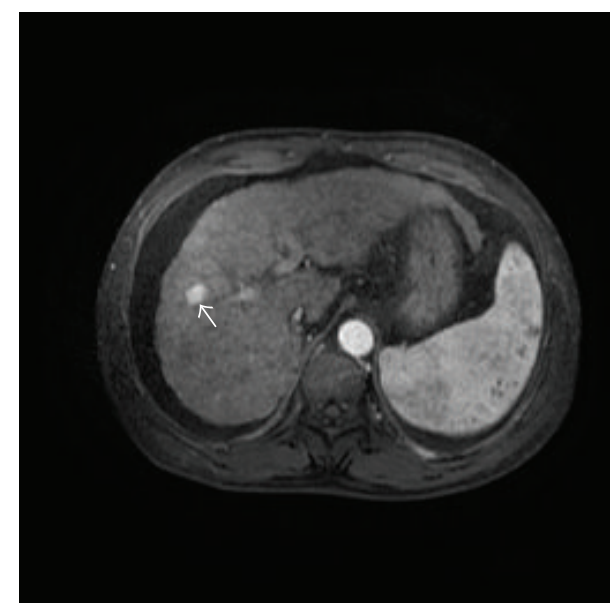

(d)

Figure 2: Transverse images in 62-year-old man with HCC. (a) Fat-suppressed T2-weighted fast spin-echo image shows that lesion is difficult to be detected. (b) DW image with higher $b$-value shows that lesion is strongly hyperintense to the liver. (c) DW image with low $b$-value shows that lesion is obscure. (d) Contrast-enhanced T1-weighted image in arterial phase demonstrates strong enhancement of lesion.

note that lesions with definite diffusion restriction can also be detected on DWI600 in left lobe. Meanwhile, results from our subgroup analysis on lesion size suggested that the detection rate was significantly influenced by the lesion size. The larger the lesion was, the easier it could be detected on all three MR sequences.

Our results were in disagreement with previous studies $[14,17]$ that DWI with a low $b$-value can significantly improve detection rate of both malignant and benign FLLs, comparing with T2-weighted imaging. For example, our study found that the detection rate of T2WI (70.8\%) in malignant solid FLLs was as much as of DWI100 (72.4\%). There is no doubt that the suppression of intrahepatic vessels with DWI100 considerably improves the detectability of perivascular lesions. However, T2WI is still helpful in detecting perivascular lesions when the size of lesion is much larger than adjacent vessel or when the lesion-to-vessel contrast is obvious. In addition, one potential explanation is that lacking the susceptibility artifact and image distortion, T2WI is helpful in detection of FLLs in the periphery of the liver and in the subphrenic hepatic areas.
There are several limitations of the study. First, the low and higher $b$-value DW images were acquired using different techniques for respiratory motion suppression and different numbers of signal averages. The results of this study may be confounded by the differences in acquisition between breath-hold and respiratory-triggered study and differences in the number of signal averages. The reason is that the MR scanners used in our study could not obtain two $b$-values DWI in one acquisition. The breath-hold acquisition mode for the DWI100 was selected to achieve a short acquisition time and few motion artifacts. In addition, the superiority of respiratory-triggered DW imaging over breath-hold DW imaging for lesion detection has been suggested by previous report [14]. Second, not all FLLs were confirmed pathologically. However, clinical diagnosis can be firmly established based on careful consensus reading by experienced abdominal radiologists and follow-up data. Third, we did not make analysis of apparent diffusion coefficient (ADC) maps, because analysis of ADC values was not within the scope of this work. Fourth, we did not investigate contrast-enhanced 


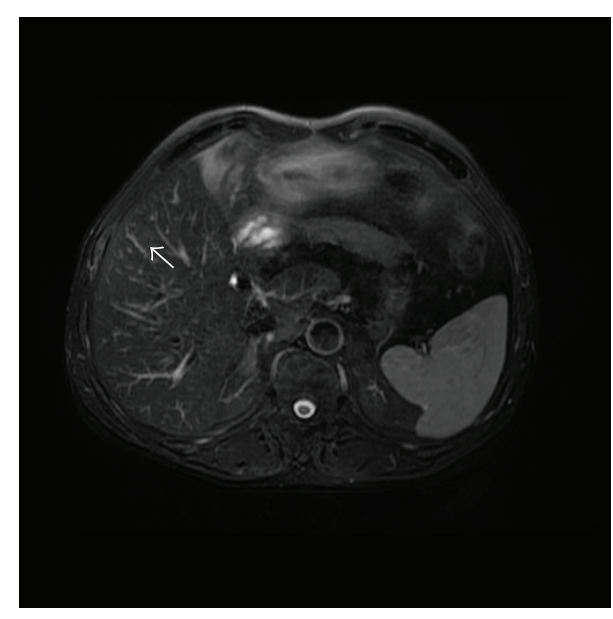

(a)

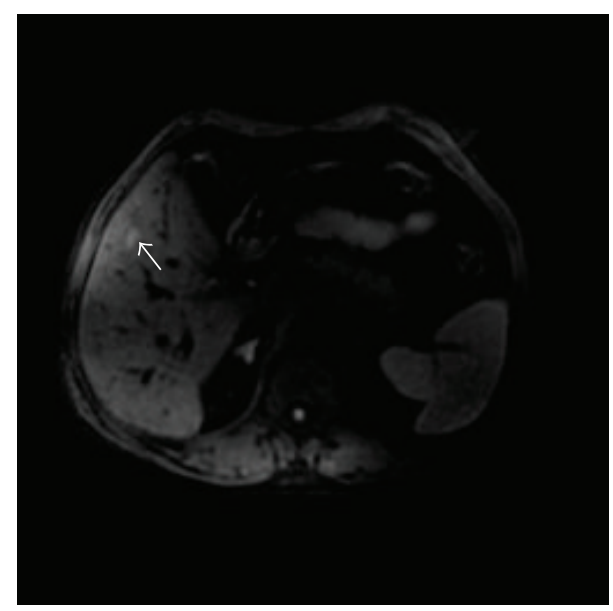

(c)

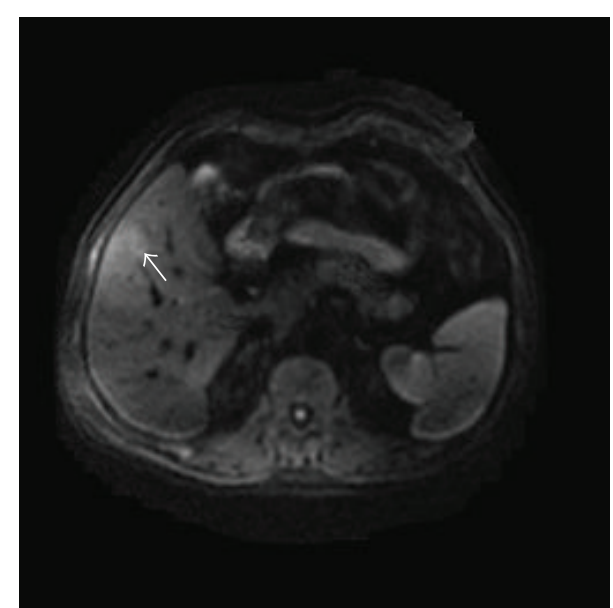

(b)

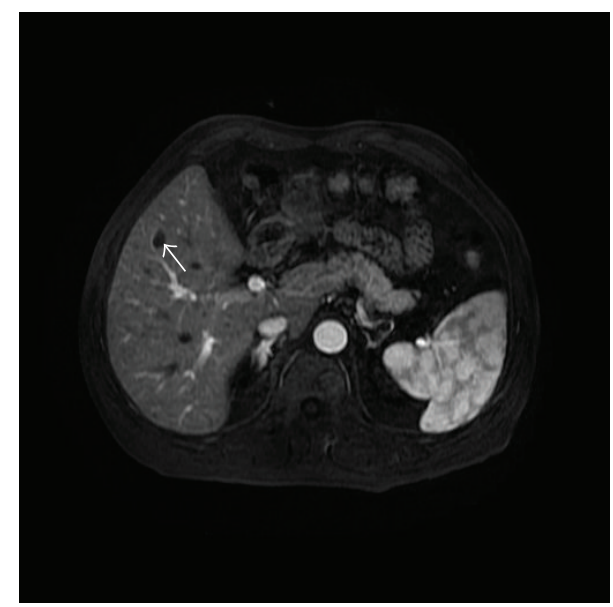

(d)

FIGURE 3: Transverse images in 56-year-old man with solitary necrotic nodule. (a) Fat-suppressed T2-weighted fast spin-echo image shows that lesion is isointense to the liver. (b) DW image with higher $b$-value shows that lesion is difficult to be identified. (c) DW image with low $b$-value shows lesion of hyperintensity could easily be detected. (d) Contrast-enhanced T1-weighted image in arterial phase demonstrates nonenhancement of lesion.

MR sequence in our study. Contrast-enhanced MR sequence is better than unenhanced MR sequences in the detection of FLLs [36]. Nevertheless, DWI and T2WI play an irreplaceable role in the detection of FLLs in patients having contrast agent allergy or renal dysfunction. Fifth, the number of benign FLLs and small FLLs $(<10 \mathrm{~mm})$ was relatively small. However, we believe our results are valid because we included a consecutive series of patients during a relatively long period and covered topic of common disease.

In conclusion, DWI600 was equivalent to DWI100 and T2WI for the detection of solid FLLs in all lesions but was significantly more accurate in detection of malignant solid FLLs and lesions larger than $10 \mathrm{~mm}$. The results of our study show the superiority of DWI600 in the detection of malignant solid FLLs, but also the disadvantage of DWI600 for the depiction of benign solid FLLs such as FNHs and IPTs and tiny lesions. Both low and higher $b$-value diffusionweighted imaging should be recommended as supplementary MR sequences in clinical practice.

\section{Conflict of Interests}

The authors declare that there is no conflict of interests regarding the publication of this paper.

\section{Authors' Contribution}

Da-wei Yang and Ke-yang Wang contributed equally to this study.

\section{Acknowledgments}

Editorial support was provided by Xiao-zheng Wang, M.D. The authors would like to express their enormous appreciation and gratitude to all participating sites. This study was supported by (1) Beijing Municipal Science \& Technology Commission, Contract Grant no. D101100050010056, and (2) National Key Technology R\&D Program, Contract Grant no. 2015BAI13B09. 


\section{References}

[1] T. Yau, V. Y. F. Tang, T.-J. Yao, S.-T. Fan, C.-M. Lo, and R. T. P. Poon, "Development of Hong Kong liver cancer staging system with treatment stratification for patients with hepatocellular carcinoma," Gastroenterology, vol. 146, no. 7, pp. 1691-1700.e3, 2014.

[2] Y.-X. J. Wáng, T. De Baere, J.-M. Idée, and S. Ballet, "Transcatheter embolization therapy in liver cancer: an update of clinical evidences," Chinese Journal of Cancer Research, vol. 27, no. 2, pp. 96-121, 2015.

[3] Y.-X. J. Wáng, Y. Choi, Z. Chen, S. Laurent, and S. L. Gibbs, "Molecular imaging: from bench to clinic," BioMed Research International, vol. 2014, Article ID 357258, 3 pages, 2014.

[4] X. Xu, L. Luo, J. Chen et al., "Acoustic radiation force impulse elastography for efficacy evaluation after hepatocellular carcinoma radiofrequency ablation: a comparative study with contrast-enhanced ultrasound," BioMed Research International, vol. 2014, Article ID 901642, 7 pages, 2014.

[5] R. Loffroy, S. Favelier, V. Cherblanc, and L. Estivalet, "C-arm dual-phase cone-beam CT: a revolutionary real-time imaging modality to assess drug-eluting beads TACE success in liver cancer patients," Quantitative Imaging in Medicine and Surgery, vol. 3, no. 4, pp. 196-199, 2013.

[6] R. Loffroy, S. Favelier, O. Chevallier et al., "Preoperative portal vein embolization in liver cancer: indications, techniques and outcomes," Quantitative Imaging in Medicine and Surgery, vol. 5, no. 5, pp. 730-739, 2015.

[7] G.-H. Kwon, K. A. Kim, S. S. Hwang et al., "Efficiency of non-contrast-enhanced liver imaging sequences added to initial rectal MRI in rectal cancer patients," PLoS ONE, vol. 10, no. 9, Article ID e0137320, 2015.

[8] T. Namimoto, M. Nakagawa, Y. Kizaki et al., "Characterization of liver tumors by diffusion-weighted imaging: comparison of diagnostic performance using the mean and minimum apparent diffusion coefficient," Journal of Computer Assisted Tomography, vol. 39, no. 4, pp. 453-461, 2015.

[9] S. E. Bouchaibi, K. Coenegrachts, M. A. Bali, J. Absil, T. Metens, and C. Matos, "Focal liver lesions detection: comparison of respiratory-triggering, triggering and tracking navigator and tracking-only navigator in diffusion-weighted imaging," European Journal of Radiology, vol. 84, no. 10, pp. 1857-1865, 2015.

[10] S. Lewis, A. Kamath, M. Chatterji et al., "Diffusion-weighted imaging of the liver in patients with chronic liver disease: comparison of monopolar and bipolar diffusion gradients for image quality and lesion detection," American Journal of Roentgenology, vol. 204, no. 1, pp. 59-68, 2015.

[11] N. Galea, V. Cantisani, and B. Taouli, "Liver lesion detection and characterization: role of diffusion-weighted imaging," Journal of Magnetic Resonance Imaging, vol. 37, no. 6, pp. 1260-1276, 2013.

[12] N. Bharwani and D. M. Koh, "Diffusion-weighted imaging of the liver: an update," Cancer Imaging, vol. 13, no. 2, pp. 171-185, 2013.

[13] T. Takahara and T. C. Kwee, "Low b-value diffusion-weighted imaging: emerging applications in the body," Journal of Magnetic Resonance Imaging, vol. 35, no. 6, pp. 1266-1273, 2012.

[14] T. Parikh, S. J. Drew, V. S. Lee et al., "Focal liver lesion detection and characterization with diffusion-weighted MR imaging: comparison with standard breath-hold T2-weighted imaging," Radiology, vol. 246, no. 3, pp. 812-822, 2008.

[15] H. Wang, X.-Y. Wang, X.-X. Jiang, and Z.-X. Ye, “Comparison of diffusion-weighted with T2-weighted Imaging for detection of small hepatocellular carcinoma in cirrhosis: preliminary quantitative study at 3-T," Academic Radiology, vol. 17, no. 2, pp. 239-243, 2010.

[16] K. Coenegrachts, J. Delanote, L. Ter Beek et al., "Improved focal liver lesion detection: comparison of single-shot diffusionweighted echoplanar and single-shot $T_{2}$ weighted turbo spin echo techniques," British Journal of Radiology, vol. 80, no. 955, pp. 524-531, 2007.

[17] C. J. Zech, K. A. Herrmann, O. Dietrich, W. Horger, M. F. Reiser, and S. O. Schoenberg, "Black-blood diffusion-weighted EPI acquisition of the liver with parallel imaging: comparison with a standard T2-weighted sequence for detection of focal liver lesions," Investigative Radiology, vol. 43, no. 4, pp. 261-266, 2008.

[18] H. K. Hussain, I. Syed, H. V. Nghiem et al., "T2-weighted MR imaging in the assessment of cirrhotic liver," Radiology, vol. 230, no. 3, pp. 637-644, 2004.

[19] J. Chen, M. Wu, R. Liu, S. Li, R. Gao, and B. Song, "Preoperative evaluation of the histological grade of hepatocellular carcinoma with diffusion-weighted imaging: a meta-analysis," PLoS ONE, vol. 10, no. 2, Article ID e0117661, 2015.

[20] S. M. Hussain, T. Terkivatan, P. E. Zondervan et al., "Focal nodular hyperplasia: findings at state-of-the-art MR imaging, US, CT, and pathologic analysis," Radiographics, vol. 24, no. 1, pp. 3-17, 2004.

[21] S. Goshima, M. Kanematsu, H. Kondo et al., "Diffusionweighted imaging of the liver: optimizing $b$ value for the detection and characterization of benign and malignant hepatic lesions," Journal of Magnetic Resonance Imaging, vol. 28, no. 3, pp. 691-697, 2008.

[22] M. Bruegel, J. Gaa, S. Waldt et al., "Diagnosis of hepatic metastasis: comparison of respiration-triggered diffusionweighted echo-planar MRI and five T2-weighted turbo spinecho sequences," American Journal of Roentgenology, vol. 191, no. 5, pp. 1421-1429, 2008.

[23] X.-Y. Cui and H.-W. Chen, "Role of diffusion-weighted magnetic resonance imaging in the diagnosis of extrahepatic cholangiocarcinoma," World Journal of Gastroenterology, vol. 16, no. 25, pp. 3196-3201, 2010.

[24] M. Ronot and V. Vilgrain, "Imaging of benign hepatocellular lesions: current concepts and recent updates," Clinics and Research in Hepatology and Gastroenterology, vol. 38, no. 6, pp. 681-688, 2014.

[25] J. Y. Park, M. S. Choi, Y.-S. Lim et al., "Clinical features, image findings, and prognosis of inflammatory pseudotumor of the liver: a multicenter experience of 45 cases," Gut and liver, vol. 8, no. 1, pp. 58-63, 2014.

[26] L. X. Wang, K. Liu, G. W. Lin, and R. Y. Zhai, "Solitary necrotic nodules of the liver: histology and diagnosis with CT and MRI," Hepatitis Monthly, vol. 12, no. 8, Article ID e6212, 2012.

[27] S. R. Prasad, H. Wang, H. Rosas et al., "Fat-containing lesions of the liver: radiologic-pathologic correlation," Radiographics, vol. 25, no. 2, pp. 321-331, 2005.

[28] R. F. Sheng, M. S. Zeng, Y. Ji, L. Yang, C. Z. Chen, and S. X. Rao, "MR features of small hepatocellular carcinoma in normal, fibrotic, and cirrhotic livers: a comparative study," Abdominal Imaging, vol. 40, no. 8, pp. 3062-3069, 2015.

[29] M. A. Fischer, D. A. Raptis, O. F. Donati et al., "MR imaging features for improved diagnosis of hepatocellular carcinoma in the non-cirrhotic liver: multi-center evaluation," European Journal of Radiology, vol. 84, no. 10, pp. 1879-1887, 2015. 
[30] J. Bruix and M. Sherman, "Management of hepatocellular carcinoma," Hepatology, vol. 42, pp. 1208-1236, 2005.

[31] A. D. Hardie, M. Naik, E. M. Hecht et al., "Diagnosis of liver metastases: value of diffusion-weighted MRI compared with gadolinium-enhanced MRI," European Radiology, vol. 20, no. 6, pp. 1431-1441, 2010.

[32] I.-M. Danet, R. C. Semelka, P. Leonardou et al., "Spectrum of MRI appearances of untreated metastases of the liver," American Journal of Roentgenology, vol. 181, no. 3, pp. 809-817, 2003.

[33] G. A. Krinsky, V. S. Lee, N. D. Theise et al., "Hepatocellular carcinoma and dysplastic nodules in patients with cirrhosis: prospective diagnosis with MR imaging and explantation correlation," Radiology, vol. 219, no. 2, pp. 445-454, 2001.

[34] F. Agnello, M. Ronot, D. C. Valla, R. Sinkus, B. E. Van Beers, and V. Vilgrain, "High-b-value diffusion-weighted MR imaging of benign hepatocellular lesions: quantitative and qualitative analysis," Radiology, vol. 262, no. 2, pp. 511-519, 2012.

[35] S. M. Hussain, J. De Becker, W. C. J. Hop, S. Dwarkasing, and P. A. Wielopolski, "Can a single-shot black-blood T2weighted spin-echo echo-planar imaging sequence with sensitivity encoding replace the respiratory-triggered turbo spinecho sequence for the liver? An optimization and feasibility study," Journal of Magnetic Resonance Imaging, vol. 21, no. 3, pp. 219-229, 2005.

[36] P. Soyer, M. Boudiaf, V. Placé et al., "Preoperative detection of hepatic metastases: comparison of diffusion-weighted, T2weighted fast spin echo and gadolinium-enhanced MR imaging using surgical and histopathologic findings as standard of reference," European Journal of Radiology, vol. 80, no. 2, pp. 245252, 2011. 


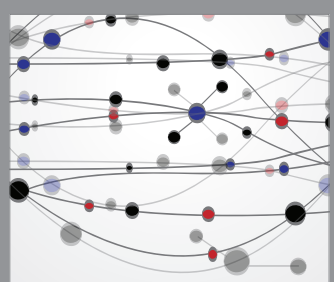

The Scientific World Journal
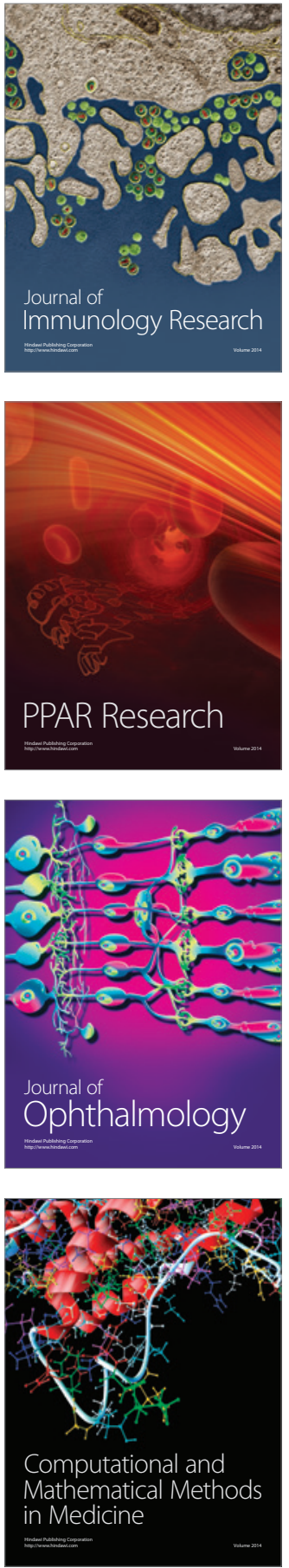

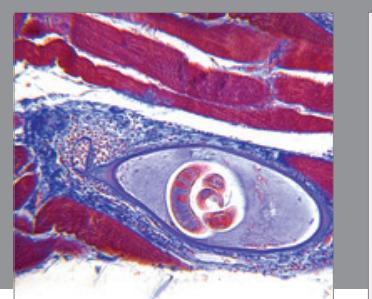

Gastroenterology Research and Practice

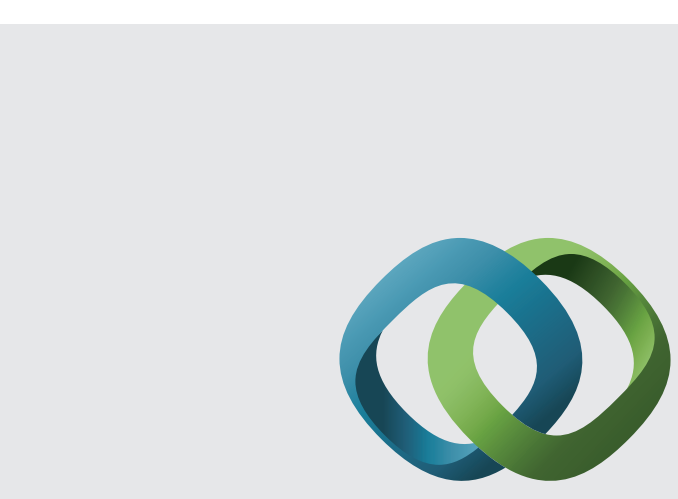

\section{Hindawi}

Submit your manuscripts at

http://www.hindawi.com
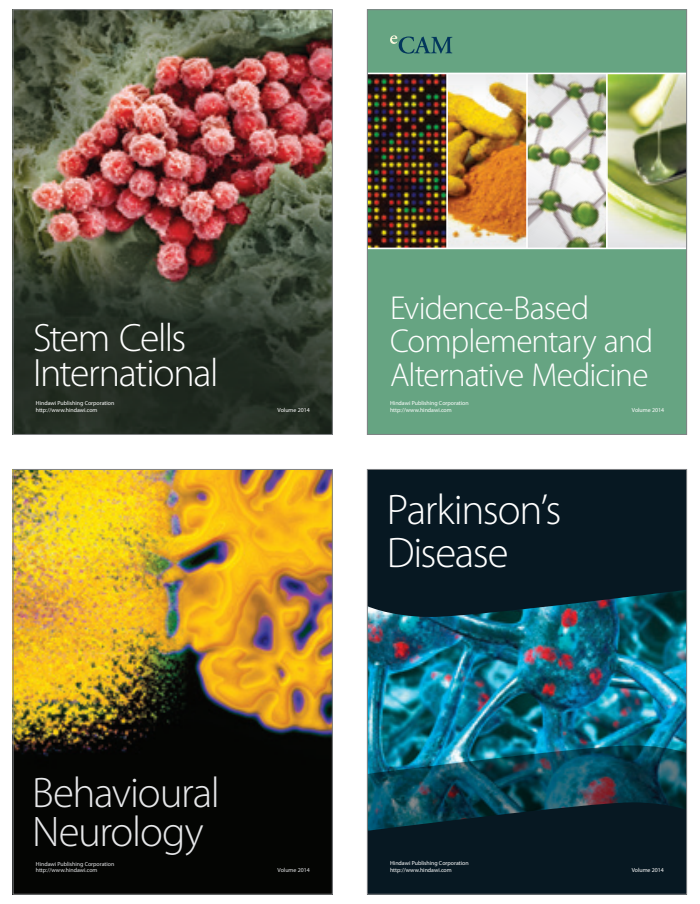
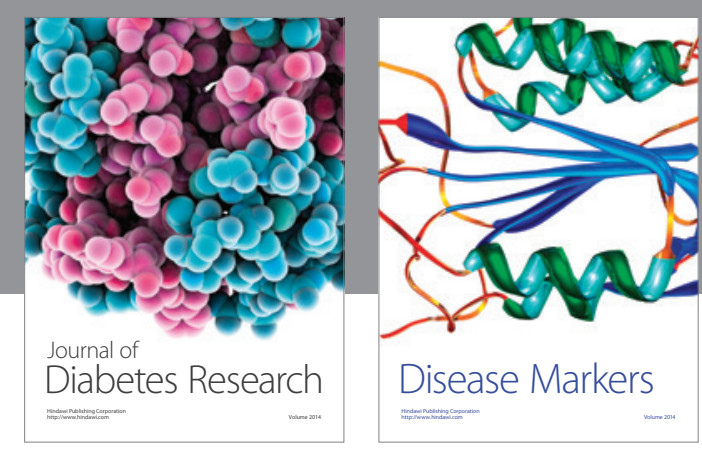

Disease Markers
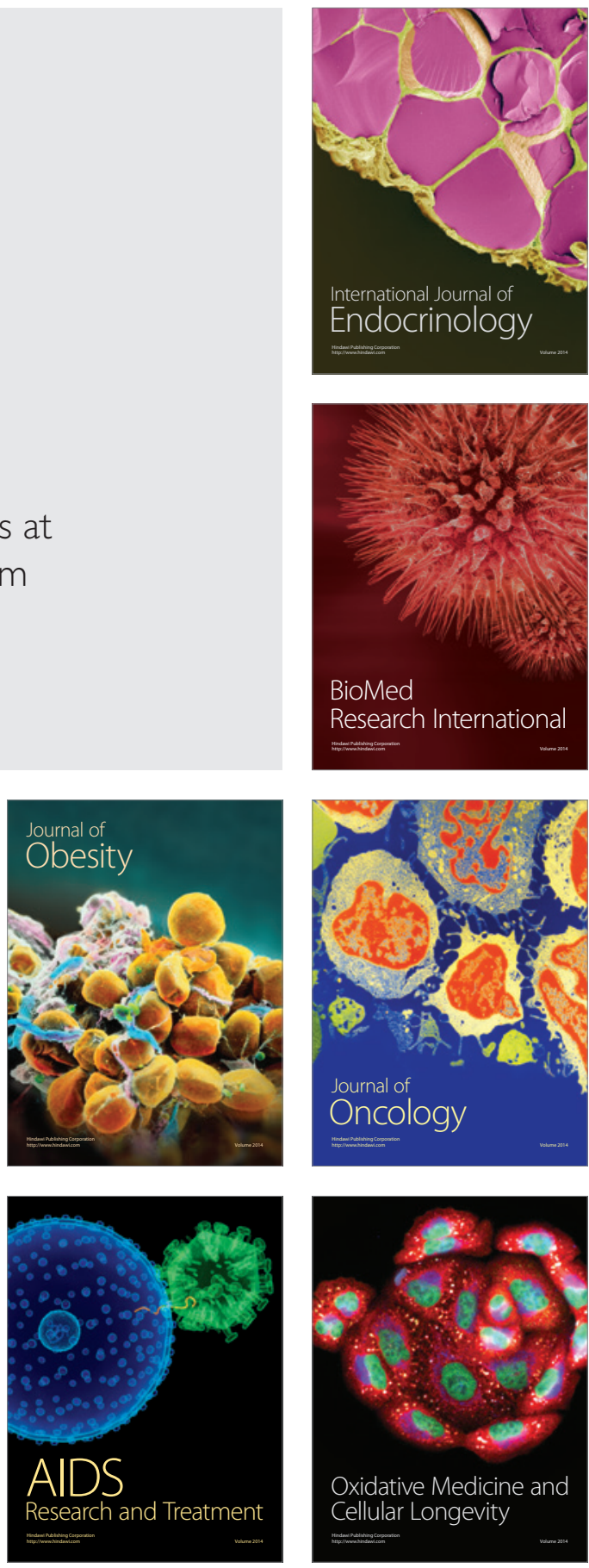